\title{
Síndrome de Pearson en un niño trasplantado debido a anemia de Diamond-Blackfan
} Pearson syndrome in a child transplanted for Diamond-Blackfan anemia

\author{
Dr. Vedat Uygun ${ }^{a}$ Dra. Hayriye Daloğlu ${ }^{a}$, Dra. Seda Öztürkmen ${ }^{b}$ Dra. Gülsün Karasu ${ }^{b}$ y Dr. Akif Yeşilipek
}

\section{RESUMEN}

El síndrome de Pearson (SP) comparte varias características con la anemia de Diamond-Blackfan (ADB), incluida la anemia grave de inicio temprano, por lo que es importante hacer un diagnóstico diferencial. El diagnóstico diferencial de la ADB y el SP es fundamental, ya que los pacientes con ADB podrían responder al tratamiento con corticoesteroides, presentar remisión o beneficiarse del trasplante de células madre hematopoyéticas (TCMH). Sin embargo, los pacientes conSP tienen un pronóstico diferente, con un riesgo muy elevado de acidosis, problemas metabólicos y disfunción pancreática, y una expectativa de vida menor en comparación con aquellos con ADB. En este artículo, presentamos el caso de un paciente sometido a TCMH para la $\mathrm{ADB}$, pero que luego fue diagnosticado con SP tras desarrollar algunas complicaciones.

Palabras clave: anemia de Diamond-Blackfan, trasplante de células madre hematopoyéticas, sindrome de Pearson.

http: / / dx.doi.org/10.5546/ aap.2021.e559

Texto completo en inglés:

http:/ / dx.doi.org/10.5546/ aap.2021.eng.e559

Cómo citar: Uygun V, Daloğlu H, Öztürkmen S, Karasu G, Yeşilipek A. Síndrome de Pearson en un niño trasplantado debido a anemia de Diamond-Blackfan. Arch Argent Pediatr 2021;119(5):e559-e561.

\section{Siglas y abreviaturas}

SP: síndrome de Pearson.

ADB: anemia de Diamond-Blackfan.

TCMH: trasplante de células madre hematopoyéticas.

EICH: enfermedad injerto contra huésped.

CsA: ciclosporina A.

NMS: no mielosupresor.

a. Facultad de Medicina de İstinye Üniversitesi, Hospital Medical Park Antalya, Departamento de Trasplante de Médula Ósea Pediátrico, Antalya, Turquía.

b. Hospital Medical Park Antalya, Departamento de

Trasplante de Médula Ósea Pediátrico, Antalya, Turquía.

Correspondencia:

Dr. Vedat Uygun: veddat@hotmail.com

Financiamiento: Ninguno.

Conflicto de intereses: Ninguno que declarar.

Recibido: 4-2-2021

Aceptado: 31-3-2021

\section{INTRODUCCIÓN}

La anemia de Diamond-Blackfan (ADB) se caracteriza por anemia macrocítica hiporregenerativa congénita grave, asociada a veces con malformaciones congénitas con retraso del crecimiento. La incidencia estimada es de 7 casos/millón de nacimientos. ${ }^{1}$ Las mutaciones en los genes de la proteína ribosómica (ribosomal protein, RP) suelen ser responsables de este trastorno. ${ }^{2}$ La base del tratamiento de la ADB son los corticoesteroides y la transfusión de sangre. El trasplante de células madre hematopoyéticas (TCMH) ha funcionado en los pacientes con resistencia a los corticoesteroides. ${ }^{3}$ El síndrome de Pearson (SP) es una afección relativamente rara (incidencia estimada de 1 caso/millón de nacimientos) ${ }^{4}$ causada por deleciones grandes en el ADN mitocondrial y que se desarrolla durante la lactancia con anemia macrocítica grave dependiente de transfusiones $y$, a menudo, asociada con distintos grados de pancitopenia, acidosis metabólica y alteración tisular.

El diagnóstico diferencial de la ADB y el SP es fundamental, ya que los pacientes con ADB podrían responder al tratamiento con corticoesteroides, presentar remisión o beneficiarse del TCMH. Sin embargo, los pacientes con SP tienen un pronóstico diferente, con un riesgo muy elevado de acidosis, problemas metabólicos y disfunción pancreática, y una expectativa de vida menor en comparación con aquellos con ADB. El tratamiento con corticoesteroides no está indicado para el SP, ya que podría aumentar el riesgo de complicaciones sin beneficios, ${ }^{5}$ y el TCMH no es uno de los tratamientos principales. ${ }^{6}$

En este artículo, presentamos el caso de un paciente sometido a $\mathrm{TCMH}$ para la $\mathrm{ADB}$, pero que luego fue diagnosticado con SP tras desarrollar algunas complicaciones.

\section{Presentación de un caso}

El paciente se presentó en un centro médico a los 2 meses de edad debido a palidez intensa. Dado que tenía anemia grave y que la 
concentración de hemoglobina era de 2,6 g/ dl, se le hizo una aspiración de médula ósea y se le diagnosticó ADB por aplasia eritrocitaria. Se inició la administración de metilprednisolona en una dosis de $2 \mathrm{mg} / \mathrm{kg}$; sin embargo, se interrumpió al cabo de 4 meses debido a que el paciente tuvo cataratas. Mientras tanto, fue posible analizar la secuencia del gen RPS19 del paciente, que representa aproximadamente el $25 \%$ de todos los pacientes con ADB. ${ }^{7} \mathrm{El}$ resultado fue normal. Durante el seguimiento, se observaron trombocitopenia y neutropenia leves. Dado que el paciente tenía un hermano donante compatible (varón de 20 años), se lo ingresó a nuestro centro a los 20 meses de edad para recibir un TCMH.

Se le administró busulfano en una dosis mielosupresora durante 4 días $(4,8 \mathrm{mg} / \mathrm{kg} /$ día), $150 \mathrm{mg} / \mathrm{m}^{2}$ de fludarabina, $10 \mathrm{mg} / \mathrm{kg}$ de tiotepa y $30 \mathrm{mg} / \mathrm{kg}$ de concentrado de inmunoglobulinas antitimocíticas (ATG-Fresenius) como tratamiento de acondicionamiento. Se lo sometió a un trasplante de células madre de la médula ósea con una dosis de $10 \times 10^{8} / \mathrm{kg}$ de células nucleadas totales y $7 \times 10^{6} / \mathrm{kg}$ de células CD34(+). Se administró profilaxis para la enfermedad injerto contra huésped (EICH) con ciclosporina A (CsA) y $10 \mathrm{mg} / \mathrm{m}^{2}$ de metotrexato los días 1,3 y 6 .

El paciente no presentó problemas en el período postrasplante, excepto por mucositis grave. Se observó prendimiento de plaquetas y neutrófilos los días 9 y 16 , respectivamente. El día 24, el paciente presentó sepsis con hipoglucemia e hiperamoniemia, y la concentración de lactato aumentó a $10 \mathrm{mmol} / 1-12 \mathrm{mmol} / 1$. En el mismo período, el paciente tuvo hiperbilirrubinemia directa debido a EICH en el hígado y se decidió cambiar la CsA por tacrolimus y agregar $2 \mathrm{mg} / \mathrm{kg}$ de metilprednisolona. Si bien su estado general mejoró gradualmente y la colestasis remitió, la concentración de lactato siguió elevada. Se consideró que el paciente tenía una enfermedad metabólica. Por lo tanto, se decidió hacer una prueba de detección de SP, que es clínicamente similar a la ADB. En el análisis del ADN separado antes del trasplante, se detectó una deleción mitocondrial, que indicaba la presencia de SP (deleción heteroplásmica con un cociente de sonda de 0,4 , que abarcaba los genes ATP6, COX3, ND3, ND4 y ND5 dentro de los límites de detección del método de amplificación de sondas dependiente de ligandos múltiples [MLPA]). Además, se revisaron retrospectivamente los extendidos de médula ósea obtenidos al momento del diagnóstico y se observó una vacuolización rara en los precursores mieloides y eritroides, lo que podría respaldar el diagnóstico de SP. El paciente recibió el alta a los 69 días para continuar con seguimiento ambulatorio. Sin embargo, debido a ciertos problemas sociales, el seguimiento se continuó en otra ciudad, y el paciente tuvo sepsis 5 meses después del TCMH. A pesar del tratamiento, empeoró rápidamente y falleció el día 167 posterior al trasplante.

\section{DISCUSIÓN}

La ADB y el SP comparten características y podrían no diferenciarse si no se conocen las similitudes al momento de las pruebas de diagnóstico iniciales, y el diagnóstico erróneo podría persistir por años. Un hallazgo importante que podría observarse durante el diagnóstico diferencial es la vacuolización de los precursores mieloides y eritroides en el SP. En el caso presentado en este artículo, cuando se observaron indicios de SP, se solicitaron los extendidos de médula ósea iniciales empleados para el diagnóstico y se observó una vacuolización en los precursores mieloides y eritroides. Cuando se evaluaron los hallazgos clínicos y de laboratorio del paciente en forma retrospectiva, se determinó que las vacuolas se interpretaron previamente como mielodisplasia y que no se consideró el SP debido a la ausencia de otros problemas clínicos aparte de la anemia.

Se puede argumentar que, en este caso, el trasplante causó el daño, el cual se había hecho debido al diagnóstico erróneo de ADB. Hasta donde sabemos, se informó sobre cuatro pacientes con SP que recibieron un TCMH, pero solo uno de ellos sobrevivió. ${ }^{8-11}$ Resulta interesante mencionar que dos de ellos se sometieron al trasplante tras el diagnóstico de ADB, como nuestro paciente, pero se les diagnosticó SP durante un estudio de investigación posterior al TCMH. Si bien no se disponía de información sobre el procedimiento utilizado para el TCMH y el seguimiento de los pacientes, ambos murieron antes de los 3 años de edad. Los otros dos pacientes presentaron prendimiento del injerto y alcanzaron recuentos sanguíneos normales. Uno de ellos se sometió a un segundo trasplante debido al rechazo y tuvo encefalopatía metabólica transitoria después de un tratamiento de acondicionamiento no mielosupresor (NMS) y profilaxis para la EICH con CsA. Este paciente tuvo varios problemas metabólicos y leucemia mielógena aguda 12 meses 
después del TCMH y falleció. El segundo paciente estaba vivo a los 3 años de seguimiento después del TCMH, para el cual recibió tratamiento NMS e infusión de sangre del cordón umbilical como fuente de las células madre. En el informe no se especificaba la profilaxis para la EICH. Cabe mencionar que el TCMH estaba asociado no solamente con una mejor hematopoyesis, sino también con la resolución de la hiperlacticemia. ${ }^{10}$ Nuestro paciente recibió tratamiento de acondicionamiento mielosupresor y no tuvo complicaciones importantes. Si bien no es posible establecer un tratamiento de acondicionamiento ideal en el SP debido a la escasez de casos, es posible concluir que no se producen toxicidad importante, incluso con la dosis mielosupresora, ni efectos secundarios graves con la profilaxis contra la EICH con CsA y metotrexato.

$\mathrm{Si}$ bien no suele recomendarse el TCMH para las complicaciones hematopoyéticas del SP, ya que existe una tendencia hacia una mejoría espontánea, todos los pacientes recibieron un trasplante simplemente porque sus afecciones hematológicas habían empeorado. .-11 $^{-11}$

Según estos casos limitados, el TCMH podría corregir el deterioro hematológico en el SP. Con base en la evidencia de los beneficios alogénicos del TCMH en los trastornos metabólicos hereditarios, surge la duda de si el TCMH sirve para los problemas metabólicos en el SP. Entre los pacientes informados, solamente uno mostró una mejoría de los problemas metabólicos. La diferencia con otros casos fue el uso de sangre del cordón umbilical como fuente de células madre y un seguimiento significativamente más prolongado. ${ }^{10}$ La sangre del cordón umbilical es menos madura que otras fuentes de células madre, y su inmadurez biológica y posibles efectos regenerativos podrían ser la causa de la mejoría de los problemas metabólicos en los tejidos no hematopoyéticos de ese paciente. Además, ese paciente recibió seguimiento durante 3 años después del TCMH, lo que es tiempo suficiente para que las células hematopoyéticas corrijan la lesión bioquímica causada por los problemas metabólicos en los órganos no hematológicos. Ninguno de los otros pacientes, incluido nuestro caso, sobrevivió lo suficiente como para observar este beneficio.
Para los pacientes con anemia congénita, se recomienda hacer pruebas de detección de ADB y SP, aunque su estado general sea adecuado y no presenten manifestaciones sindrómicas. El trasplante de médula ósea podría ser una opción de tratamiento para el SP, en especial para los pacientes con empeoramiento de la hematopoyesis. Sigue siendo esencial hacer un diagnóstico correcto para garantizar un seguimiento adecuado y brindar tratamiento sintomático adicional.

\section{REFERENCIAS}

1. Willig TN, Niemeyer CM, Leblanc $\mathrm{T}$, Tiemann $\mathrm{C}$, et al. Identification of new prognosis factors from the clinical and epidemiologic analysis of a registry of 229 Diamond-Blackfan anemia patients. DBA group of Societe d'Hematologie et d'Immunologie Pediatrique (SHIP), Gesellshaft fur Padiatrische Onkologie und Hamatologie (GPOH), and the European Society for Pediatric Hematology and Immunology (ESPHI). Pediatr Res. 1999; 46(5):553-61.

2. Da Costa L, O'Donohue MF, van Dooijeweert B, AlbrechtK, et al. Molecular approaches to diagnose Diamond-Blackfan anemia: The EuroDBA experience. Eur J Med Genet. 2018; 61(11):664-73.

3. Fagioli F, Quarello P, Zecca M, Lanino E, et al. Haematopoietic stem cell transplantation for Diamond Blackfan anaemia: a report from the Italian Association of Paediatric Haematology and Oncology Registry. $\mathrm{Br} \mathrm{J}$ Haematol. 2014; 165(5):673-81.

4. Farruggia P, Di Cataldo A, Pinto RM, Palmisani E, et al. Pearson Syndrome: A Retrospective CohortStudy from the Marrow Failure Study Group of A.I.E.O.P. (Associazione Italiana Emato-Oncologia Pediatrica). JIMD Rep. 2016; 26:37-43.

5. Finsterer J, Frank M. Glucocorticoids for mitochondrial disorders. Singapore Med J. 2015; 56(2):122-3.

6. FarruggiaP, DiMarco F, DufourC. Pearson syndrome. Expert Rev Hematol. 2018; 11(3):239-46.

7. Willig TN, Draptchinskaia N, Dianzani I, Ball S, et al. Mutations in ribosomal protein $S 19$ gene and diamond

8. Blackfan anemia: wide variations in phenotypic expression. Blood. 1999; 94(12):4294-306.

9. Gagne KE, Ghazvinian R, Yuan D, Zon RL, et al. Pearson marrow pancreas syndrome in patients suspected to have Diamond-Blackfan anemia. Blood. 2014; 124(3):437-40.

10. Tumino M, Meli C, Farruggia P, La Spina M, et al. Clinical manifestations and management of four children with Pearson syndrome. Am JMed Genet A. 2011;155A(12):3063-6.

11. Hoyoux C, Dresse MF, Robinet S, Forget P, et al. Cord blood transplantation in a child with Pearson's disease. Pediatr Blood Cancer. 2008; 51(4):566.

12. Faraci M, Cuzzubbo D, Micalizzi C, Lanino E, et al. Allogeneic bone marrow transplantation for Pearson's syndrome. Bone Marrow Transplant. 2007; 39(9):563-5. 